\title{
OTIMIZAÇÃO MULTIVARIADA DE METODOLOGIA PARA DIGESTÃO DE MICROPARTÍCULAS POLIMÉRICAS CARREADORAS DE CÁTIONS METÁLICOS
}

\author{
Júnior O. Chagas, Hércules G. L. de Sousa, Isabela C. M. Cunha, Roberta E. S. Froes-Silva, Gilmare A. da Silva* e Fabiana \\ A. Lobo \\ Departamento de Química, Instituto de Ciências Exatas e Biológicas, Universidade Federal de Ouro Preto, 35400-000 Ouro Preto \\ - MG, Brasil
}

Recebido em 28/03/2016, aceito em 06/07/2016, publicado na web em 16/08/2016

\begin{abstract}
MULTIVARIATE OPTIMIZATION OF METHODOLOGY FOR POLYMERIC MICROPARTICLE DIGESTION METALLIC CATION CARRIERS. There are not reports in the literature of digestion processes of metallic cation carrier microparticles. Multivariate experimental designs were utilized to ensure an appropriate digestion for further analysis by atomic absorption spectroscopy. It was used a heater plate and the polimeric degradation was evaluated utilizing an analyzer of total organic carbon (TOC). For the total carbon (TC) content, the full factorial design $2^{4}$ pointed the significance of sample volume $\left(\mathrm{V}_{\mathrm{Am}}\right)$, in relation to the variables acid volume $\left(\mathrm{V}_{\mathrm{Ac}}\right)$, temperature $(\mathrm{T})$ and digestion time $(\mathrm{t})$. However, according to the normal distribution graph it was noted a possible significance of the $T$ and $t$ effects. The same was observed for TOC, including the $V_{A m} \times V_{A c} \times T$ effect suggestive of complex behavior. In the central composite design all variables were again studied and the $\mathrm{V}_{\mathrm{AM}}$ was significant, promoting a TC decreasing at the lower evaluated level. By ANOVA a quadratic model without lack of fit was found, with the significant quadratic term. The best digestion condition was: $5.00 \mathrm{~mL}$ of sample, $10.00 \mathrm{~mL}$ of nitric acid, $60{ }^{\circ} \mathrm{C}$ and $90 \mathrm{~min}$. The multivariate optimization allowed an efficient digestion, with the initial carbon concentration of $4.60 \mathrm{mg} \mathrm{L}^{-1}$ decreased to $0.55 \mathrm{mg} \mathrm{L}^{-1}$.
\end{abstract}

Keywords: metallic cations; polymeric microparticles; sample preparation; digestion; multivariate experimental designs.

\section{INTRODUÇÃO}

Os cátions metálicos são parte essencial dos fertilizantes, pois eles são responsáveis por várias funções nas plantas, que vão desde a participação na síntese de proteínas, até sua presença em enzimas que participam de processos da fotossíntese. ${ }^{1-2}$ Fertilizantes ou adubos são compostos químicos inorgânicos ou orgânicos utilizados para propiciar os nutrientes essenciais ao desenvolvimento das plantas, e são aplicados na agricultura para um maior rendimento da produção. ${ }^{3}$ No entanto, atualmente, estudos ${ }^{4-5}$ demonstram que para a maioria dos fertilizantes usados, parte dos seus nutrientes são perdidos no ambiente, podendo ocasionar uma série de contaminações ambientais. Por exemplo, os fertilizantes que possuem em sua composição nitratos e fosfatos podem perder parte desses compostos em processos de lixiviação, propiciando o processo de eutrofização em águas de mananciais e lençóis freáticos. Ainda, os fertilizantes quando não aplicados de forma correta podem proporcionar desequilíbrios no ecossistema e aumento do número das fontes de toxicidade aguda e carcinogênicas para os seres humanos. ${ }^{4-5}$ Desta forma, o uso adequado dos fertilizantes é uma atividade que deve ser levada em consideração, visando à sustentabilidade econômica, ambiental e social do agronegócio mundial.

Nos últimos anos a tecnologia de liberação controlada/modificada tem ganhado destaque, pois ela emergiu como uma importante alternativa capaz de resolver alguns dos problemas que acompanham o uso de substâncias ativas de interesse em alguns setores, ${ }^{6}$ como por exemplo o agrícola (pesticidas e herbicidas), ${ }^{7-11}$ alimentício, ${ }^{12-13} \mathrm{o}$ farmacêutico, ${ }^{14-17} \mathrm{e}$, ineditamente, poderá ser aplicada para os cátions metálicos essenciais às plantas visando o aumento da eficiência dos fertilizantes, ou seja, aumento da disponibilidade destes no solo, minimização dos impactos ambientais e garantia de um desenvolvimento mundial considerado sustentável. Esta tecnologia propicia a liberação dos princípios ativos no decorrer de um determinado tempo através

*e-mail: gilmare@iceb.ufop.br de processos físico-químicos, tais como dessorção e/ou erosão, sendo capaz de permitir a alteração das propriedades físico-químicas dos ativos para a liberação controlada/adequada destes, ${ }^{6,18}$ possibilitando a atuação de forma contínua e prolongada dos cátions metálicos.

Existem vários parâmetros utilizados para a avaliação da eficiência destes sistemas. Um deles é o cálculo da taxa de encapsulamento dos nutrientes na matriz polimérica, como também a concentração de nutriente que este sistema libera no decorrer de um determinado tempo. ${ }^{6-18}$ Assim, são requeridas técnicas analíticas para a quantificação da concentração dos cátions metálicos nesses processos.

A determinação de cátions metálicos, nas mais diferentes matrizes, é tradicionalmente realizada utilizando-se técnicas espectrométricas, em função de sua exatidão, precisão e relativa simplicidade de manuseio. No entanto, amostras que são analisadas por tais técnicas requerem um preparo adequado para possibilitar a detecção e eliminação de possíveis interferentes. ${ }^{19}$ Portanto, metodologias para a abertura de amostras dos sistemas de liberação controlada são necessárias e essenciais para a quantificação correta de metais e o não comprometimento dos equipamentos e resultados obtidos.

Atualmente existem vários métodos de preparo para diferentes tipos de amostras. Especialmente para matrizes poliméricas são relatados alguns na literatura. ${ }^{20-22}$ Porém, aberturas de amostras de sistemas de liberação controlada poliméricos são inexistentes.

Técnicas de preparo de amostras para análise por absorção atômica devem apresentar simplicidade, rapidez, utilizar pequenos volumes de ácidos, permitir a dissolução de grande número de amostras e, finalmente, produzir resultados precisos e exatos. ${ }^{19} \mathrm{O}$ sistema tradicional usado é a digestão de amostras com ácido em chapa aquecedora e agitação mecânica, pois é relativamente simples e barato. No entanto, existem muitas variáveis envolvidas neste procedimento que podem interferir diretamente no ótimo do processo, tais como: tipo e volume de ácido empregado, temperatura, tempo de digestão, entre outras. Portanto, estudos que demonstrem a condição ótima para a abertura das partículas poliméricas são fundamentais. 
Com isso, torna-se muito útil e mesmo necessário o uso de experimentos estatisticamente planejados de forma multivariada para a investigação do real efeito das possíveis variáveis, assim como das interações entre elas, sobre o sistema acima referenciado. Quando se trabalha com um sistema complexo em que muitas variáveis são capazes de influenciar, porém não se sabe em qual magnitude, pode-se fazer uso como primeira etapa do processo de investigação/otimização de um planejamento de triagem. Fatores significativos selecionados após execução de um planejamento adequado de triagem podem ser empregados em uma metodologia de superfície de resposta (RSM), para a otimização do experimento. ${ }^{23-25}$

Uma vez que na literatura é inexistente o relato de condições ótimas para abertura de amostras de partículas poliméricas de processos de liberação controlada carreadoras de cátions metálicos, este trabalho teve como objetivo desenvolver uma metodologia, de fácil aplicação e baixo gasto de reagentes, para a digestão destas matrizes e posterior quantificação de íons metálicos por espectroscopia de absorção atômica por chama (FAAS), com o auxílio de métodos experimentais de otimização multivariada.

\section{PARTE EXPERIMENTAL}

\section{Reagentes, soluções e amostras}

Todas as soluções foram preparadas com reagentes de grau analítico e os volumes finais ajustados com água deionizada do sistema Milli- ${ }^{\circledR}$ (Millipore Corporation). Todas as vidrarias foram previamente lavadas em banhos contendo ácido nítrico $\left(\mathrm{HNO}_{3}\right)$ (Synth) $10 \%$ v/v por $24 \mathrm{~h}$. As soluções de referência de carbono total e inorgânico foram preparadas pela dissolução de $2,1250 \mathrm{~g}$ de hidrogenoftalato de potássio $\left(\mathrm{C}_{8} \mathrm{H}_{5} \mathrm{KO}_{4}\right)$ (Sigma Aldrich) em $1 \mathrm{~L}$ de água deionizada para a primeira, e dissolução de 3,4970 g de hidrogenocarbonato de sódio $\left(\mathrm{NaHCO}_{3}\right)$ (Sigma Aldrich) em $1 \mathrm{~L}$ de água deionizada para a segunda. Já as soluções dos cátions metálicos de cobre, ferro, magnésio, manganês, potássio e zinco foram preparadas com diluições seriais de padrões de $999 \pm 4 \mathrm{mg} \mathrm{L}^{-1}$ (Sigma Aldrich). Todas as soluções foram armazenadas e mantidas refrigeradas a $8{ }^{\circ} \mathrm{C}$ para preservação.

O preparo dos sistemas poliméricos foi realizado segundo o método de nanoprecipitação, que consiste na precipitação ou deposição interfacial de um polímero pré-formado na interface de uma emulsão óleo/água (O/A), promovendo a formação dos sistemas carreadores. ${ }^{26}$ Inicialmente, pesou-se aproximadamente 5,0000 g de álcool polivinílico (PVA) Sigma Aldrich e procedeu-se a sua solubilização em água deionizada a $70^{\circ} \mathrm{C}$ sob agitação magnética com uso de agitador magnético Hot Lab II - Nalgon, para o preparo de uma solução de PVA $5 \% \mathrm{~m} / \mathrm{v}$. Posteriormente, realizou-se diluições das soluções de referência dos cátions metálicos. Alíquotas destas soluções foram adicionadas a $100 \mathrm{~mL}$ da solução de PVA.

Para o preparo da solução orgânica foram pesados aproximadamente $600,0 \mathrm{mg}$ de poli(E-caprolactona) - Sigma Aldrich - e $300,0 \mathrm{mg}$ de um polímero obtido de rejeito industrial $(70 \% \mathrm{~m} / \mathrm{m})-$ Verti Ecotecnologias UFMG, que foram solubilizados, sob agitação mecânica e temperatura de $70{ }^{\circ} \mathrm{C}$, em uma mistura de água-clorofórmio (Sigma Aldrich) na proporção 1:3. Em seguida, essa solução foi adicionada à solução de PVA com os cátions metálicos, onde foi mantida sob agitação e temperatura de $70{ }^{\circ} \mathrm{C}$ por $4 \mathrm{~h}$. A emulsão foi mantida em capela por 24 h para a evaporação do solvente orgânico. No fim deste processo, completou-se o volume para $200 \mathrm{~mL}$ com água deionizada, onde metade desta suspensão foi filtrada com papel filtro quantitativo JP 40 faixa branca. Os materiais retidos no papel de filtro do procedimento citado foram armazenados e mantidos em dessecador. Após completamente seca, certa quantidade de amostra foi coletada e posta sob uma fita de carbono. As amostras foram levadas a um metalizador Q150R S Aname para a deposição de ouro (100 a 200 nm) sob vácuo. Em seguida, a análise da morfologia das amostras por meio das partículas metalizadas foi feita utilizando um microscópio eletrônico de varredura (MEV), Jeol 1200.

Para a digestão das amostras foi utilizado um agitador magnético Hot Lab II NalgonAs, $\mathrm{HNO}_{3}$ concentrado e termômetro químico $\left(-10+110: 1{ }^{\circ} \mathrm{C}-260 \mathrm{~mm}\right.$, Incoterm) que foi preso ao sistema por um suporte universal conectado à chapa. Posteriormente, as soluções de cada ensaio dos planejamentos experimentais utilizados, conforme as designações descritas mais adiante, foram filtradas em sistema de filtração acoplado a uma bomba a vácuo NOF-650 - Max Pump e membrana de filtração $0,45 \mu \mathrm{m}$ - Merck, para a posterior análise da concentração de carbono orgânico total (TC) e carbono inorgânico (IC).

\section{Instrumentação}

Para a avaliação da digestão das amostras poliméricas utilizou-se um analisador de carbono orgânico total TOC-L - Shimadzu, controlado pelo software TOC Control-V Shimadzu ${ }^{\circledR}$, com gás de arraste oxigênio $\left(\mathrm{O}_{2}\right)$ com grau de pureza analítica ( $\left.>99,5 \%\right)$ (White Martins) e os dados foram analisados através do software TOC Control-V Shimadzu. Foram analisados os teores de carbono orgânico, inorgânico e total residuais nos ensaios gerados pelos planejamentos experimentais multivariados empregados no desenvolvimento do método, apresentados a seguir.

\section{Otimização multivariada}

\section{Triagem}

A definição dos níveis nos quais as variáveis foram estudadas na triagem se deu a partir de testes preliminares em laboratório e avaliação da literatura disponível para as amostras mais similares às deste estudo, a fim de verificar as faixas operacionais de trabalho.

Após definidas as variáveis e os respectivos níveis a serem estudados na triagem, realizou-se um planejamento fatorial completo $2^{4}$ com triplicata no ponto central, onde se identificou as variáveis que realmente possuem efeito significativo sobre o sistema de digestão das matrizes poliméricas, para um estudo mais detalhado na metodologia de superfície de resposta. Assim, as variáveis independentes investigadas foram volume de amostra, volume de ácido nítrico, temperatura e tempo de digestão. A Tabela 1 apresenta as variáveis e os respectivos níveis avaliados nesse processo.

Tabela 1. Variáveis e níveis utilizados para o planejamento fatorial completo $2^{4}$ no estudo da digestão de micropartículas de polímeros biodegradáveis carreadoras de cátions metálicos. Os valores $-1,0 \mathrm{e}+1$ representam os níveis codificados. $\mathrm{V}_{\mathrm{am}}=$ volume de amostra, $\mathrm{V}_{\mathrm{Ac}}=$ volume de ácido, $\mathrm{T}=$ temperatura $\mathrm{e} \mathrm{t}=$ tempo

\begin{tabular}{lccc}
\hline Variáveis & \multicolumn{3}{c}{ Níveis } \\
\hline $\mathrm{V}_{\mathrm{am}}(\mathrm{mL})$ & $10,00(-1)$ & $15,00(0)$ & $20,00(+1)$ \\
$\mathrm{V}_{\mathrm{Ac}}(\mathrm{mL})$ & $10,00(-1)$ & $20,00(0)$ & $40,00(+1)$ \\
$\mathrm{T}\left({ }^{\circ} \mathrm{C}\right)$ & $30(-1)$ & $50(0)$ & $70(+1)$ \\
$\mathrm{t}(\min )$ & $60(-1)$ & $90(0)$ & $120(+1)$ \\
\hline
\end{tabular}

Utilizou-se planilhas eletrônicas ${ }^{23}$ para gerar a codificação dos experimentos a serem realizados, levando-se em consideração as combinações entre os níveis propostos, e para o tratamento estatístico dos resultados.

Para a análise de TOC uma curva analítica padrão foi construída. Esta foi preparada com a diluição serial de soluções padrões de 
carbono total (1000 mg L-1) e carbono inorgânico $\left(1000 \mathrm{mg} \mathrm{L}^{-1}\right)$. Tal diluição foi realizada pelo próprio equipamento, o analisador de carbono orgânico total. Todos os ensaios realizados no planejamento de triagem foram aferidos para $100 \mathrm{~mL}$. Em seguida todas as soluções foram diluídas 100 vezes e analisadas no analisador de carbono. $\mathrm{O}$ analisador de carbono orgânico total possibilita a resposta de três valores de concentração: carbono total, carbono orgânico total e carbono inorgânico, onde o TOC é diferença entre TC e IC.

Com isso foram selecionadas as condições que geravam os menores valores de sinal analítico em termos de concentração de carbono, tendo sido possível identificar as variáveis significativas e os níveis que foram estudados na metodologia de superfície de resposta.

\section{Metodologia de superfície de resposta}

O planejamento composto central (CCD) foi utilizado neste estudo para a etapa de otimização do processo de digestão das micropartículas. As variáveis e os níveis avaliados estão descritos na Tabela 2.

Tabela 2. Variáveis e os respectivos níveis avaliados no procedimento de construção de superfície de resposta para a digestão de micropartículas poliméricas carreadoras de cátions metálicos. Em parênteses são apresentados os níveis codificados. $\mathrm{V}_{\mathrm{am}}=$ volume de amostra, $\mathrm{V}_{\mathrm{Ac}}=$ volume de ácido, $\mathrm{T}=$ temperatura e $\mathrm{t}=$ tempo

\begin{tabular}{lccccc}
\hline Variáveis & \multicolumn{5}{c}{ Níveis } \\
\hline $\mathrm{V}_{\mathrm{am}}(\mathrm{mL})$ & $5,00(-2)$ & $7,50(-1)$ & $10,00(0)$ & $12,50(+1)$ & $15,00(+2)$ \\
$\mathrm{V}_{\mathrm{Ac}}(\mathrm{mL})$ & $5,00(-2)$ & $7,50(-1)$ & $10,00(0)$ & $12,50(+1)$ & $15,00(+2)$ \\
$\mathrm{T}\left({ }^{\circ} \mathrm{C}\right)$ & $40(-2)$ & $50(-1)$ & $60(0)$ & $70(+1)$ & $80(+2)$ \\
$\mathrm{t}(\mathrm{min})$ & $30(-2)$ & $60(-1)$ & $90(0)$ & $120(+1)$ & $150(+2)$ \\
\hline
\end{tabular}

Utilizou-se planilhas eletrônicas ${ }^{23}$ para a geração dos ensaios do planejamento composto central e tratamento dos dados. Todos os ensaios foram analisados pelo analisador de carbono orgânico total conforme descrito na etapa de triagem. Com este estudo foi possível definir a condição ótima de digestão das matrizes poliméricas de sistemas microestruturados carreadores de cátions metálicos para liberação controlada, como se segue.

\section{RESULTADOS E DISCUSSÃO}

\section{Matriz polimérica para liberação controlada de cátions metálicos}

As análises de MEV dos materiais obtidos a partir da nanoprecipitação possibilitaram observar a formação de micropartículas esféricas e rugosas com o diâmetro médio de $3,8 \mu \mathrm{m}$, classificando-as como micropartículas e sem a presença de formação de agregados. Para posteriores determinações dos teores dos elementos metálicos encapsulados é necessário o estabelecimento de um sistema de digestão eficiente das micropartículas, o que será apresentado nos itens a seguir.

\section{Otimização do sistema}

Os resultados da triagem mostraram que dentre todas as variáveis estudadas, somente o volume de amostra teve efeito significativo sobre o sistema de avaliação da concentração de TOC ( $p=0,0080)$ e de TC $(p=0,0037)$, diminuindo o teor de carbono das amostras com o menor nível estudado $(10,00 \mathrm{~mL})$ - efeito negativo. Para o IC, nenhuma variável foi apontada como significativa. Os valores de concentração obtidos estão apresentados na Tabela 3 .

Analisando a influência do $\mathrm{V}_{\mathrm{am}}$, pôde-se observar que esta variável foi significativa para as concentrações de TC e TOC, com as melhores respostas em 10,00 $\mathrm{mL}$. Devido à matriz polimérica possuir caráter hidrofóbico (PLC), a sua dissolução em meio ácido pode se tornar onerosa pelo aparecimento de resíduos sólidos sobre a superfície do digerido, ou ainda o material pode ser só parcialmente digerido, formando uma escória sobre as paredes das vidrarias utilizadas ${ }^{19}$. Assim, uma maior quantidade de agente oxidante (ácido nítrico neste

Tabela 3. Resultados (concentração em $\mathrm{mg} \mathrm{L}^{-1}$ de carbono) dos ensaios experimentais do planejamento fatorial completo $2^{4}$, com três replicatas no ponto central (ensaios 17 a 19), para a otimização das condições experimentais da digestão de micropartículas poliméricas: volume de amostra $\left(\mathrm{V}_{\mathrm{am}} / \mathrm{mL}\right)$, volume de ácido $\left(\mathrm{V}_{\mathrm{Ac}} / \mathrm{mL}^{-1}\right)$, temperatura $\left(\mathrm{T} /{ }^{\circ} \mathrm{C}\right)$ e tempo de digestão $(\mathrm{t} / \mathrm{min})$. Os valores em parênteses representam os níveis decodificados. As três últimas colunas correspondem aos valores de concentração de carbono total ( $\mathrm{TC} / \mathrm{mg} \mathrm{L}^{-1}$ ), carbono inorgânico (IC / $\mathrm{mg} \mathrm{L}^{-1}$ ) e carbono orgânico total (TOC) (mg L ${ }^{-1}$ ) obtidos para cada ponto experimental do planejamento

\begin{tabular}{|c|c|c|c|c|c|c|c|}
\hline Ensaio & $\mathbf{V}_{\mathrm{am}}$ & $\mathbf{V}_{\mathrm{Ac}}$ & $\mathbf{T}$ & $\mathbf{t}$ & TC & IC & TOC \\
\hline 1 & $-1(10,0)$ & $-1(10,0)$ & $-1(30,0)$ & $-1(60,0)$ & 2,0960 & 0,2599 & 2,6465 \\
\hline 2 & $+1(20,0)$ & $-1(10,0)$ & $-1(30,0)$ & $-1(60,0)$ & 4,6270 & 0,2809 & 4,3461 \\
\hline 3 & $-1(10,0)$ & $+1(40,0)$ & $-1(30,0)$ & $-1(60,0)$ & 2,7920 & 0,2743 & 2,5177 \\
\hline 4 & $+1(20,0)$ & $+1(40,0)$ & $-1(30,0)$ & $-1(60,0)$ & 4,7720 & 0,3024 & 4,4696 \\
\hline 5 & $-1(10,0)$ & $-1(10,0)$ & $+1(70,0)$ & $-1(60,0)$ & 2,5130 & 0,2186 & 2,2947 \\
\hline 6 & $+1(20,0)$ & $-1(10,0)$ & $+1(70,0)$ & $-1(60,0)$ & 3,7320 & 0,2117 & 3,5203 \\
\hline 7 & $-1(10,0)$ & $+1(40,0)$ & $+1(70,0)$ & $-1(60,0)$ & 1,9250 & 0,1915 & 1,7332 \\
\hline 8 & $+1(20,0)$ & $+1(40,0)$ & $+1(70,0)$ & $-1(60,0)$ & 3,9880 & 0,1746 & 3,8131 \\
\hline 9 & $-1(10,0)$ & $-1(10,0)$ & $-1(30,0)$ & $+1(120,0)$ & 2,0940 & 0,2521 & 1,8419 \\
\hline 10 & $+1(20,0)$ & $-1(10,0)$ & $-1(30,0)$ & $+1(120,0)$ & 4,0150 & 0,2639 & 3,7515 \\
\hline 11 & $-1(10,0)$ & $+1(40,0)$ & $-1(30,0)$ & $+1(120,0)$ & 2,3950 & 0,1472 & 2,2478 \\
\hline 12 & $+1(20,0)$ & $+1(40,0)$ & $-1(30,0)$ & $+1(120,0)$ & 3,9120 & 0,4183 & 3,4934 \\
\hline 13 & $-1(10,0)$ & $-1(10,0)$ & $+1(70,0)$ & $+1(120,0)$ & 1,6730 & 0,3146 & 1,3584 \\
\hline 14 & $+1(20,0)$ & $-1(10,0)$ & $+1(70,0)$ & $+1(120,0)$ & 3,4580 & 0,1472 & 3,3108 \\
\hline 15 & $-1(10,0)$ & $+1(40,0)$ & $+1(70,0)$ & $+1(120,0)$ & 2,1800 & 0,2004 & 1,9799 \\
\hline 16 & $+1(20,0)$ & $+1(40,0)$ & $+1(70,0)$ & $+1(120,0)$ & 4,1640 & 0,2408 & 3,9232 \\
\hline 17 & $0(15,0)$ & $0(20,0)$ & $0(50,0)$ & $0(90,0)$ & 3,1220 & 0,2027 & 2,9373 \\
\hline 18 & $0(15,0)$ & $0(20,0)$ & $0(50,0)$ & $0(90,0)$ & 3,1900 & 0,1589 & 2,9867 \\
\hline 19 & $0(15,0)$ & $0(20,0)$ & $0(50,0)$ & $0(90,0)$ & 2,7650 & 0,3469 & 2,4185 \\
\hline
\end{tabular}


trabalho) torna-se necessário para a dissolução dos polímeros. Dessa forma, pode-se entender que a diminuição do volume de amostra observada na triagem está diretamente relacionada com concentração de carbono total no processo de digestão desta.

No entanto, ao avaliar-se o gráfico de probabilidade normal para os valores de carbono total (Figura 1) observou-se que os efeitos das variáveis 3 e 4 (temperatura e tempo de digestão, respectivamente) mostraram uma possível tendência de não distribuição normal em relação às demais variáveis e suas interações. Observou-se um comportamento similar para os valores de carbono orgânico total com a adição de um efeito possivelmente não distribuído normalmente pela interação das variáveis 1,2 e 4 (volume de amostra, volume de ácido e temperatura, respectivamente), como ilustrado na Figura 2. O mesmo não aconteceu para a concentração de carbono inorgânico, já que neste caso as variáveis mostraram-se também não significativas no gráfico de probabilidade normal.

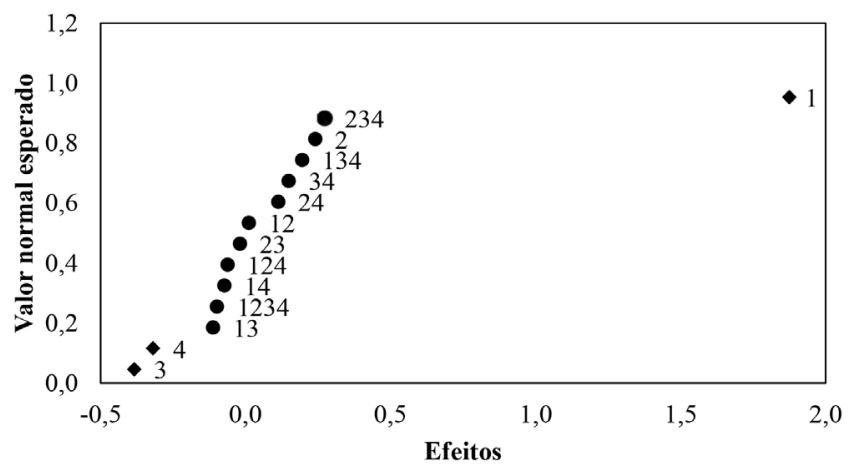

Figura 1. Gráfico de probabilidade normal para a concentração de carbono total em relação às variáveis estudadas na digestão de micropartículas poliméricas

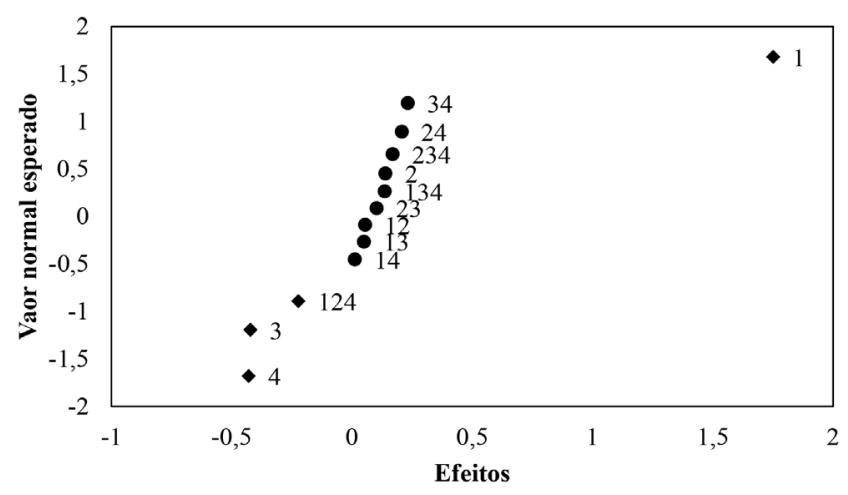

Figura 2. Gráfico de probabilidade normal para a concentração de carbono orgânico total em relação às variáveis estudadas na digestão de micropartículas poliméricas

Em regra, preparos de amostras que empregam a dissolução de matrizes com carga orgânica utilizam maiores volumes de ácidos concentrados. O ácido nítrico possui caráter oxidante e, por este fato, é comumente utilizado em digestões via úmida. Esta característica permite a quebra das ligações entre carbono e hidrogênio na maioria dos casos, o que origina uma alta eficiência da degradação da matéria orgânica ${ }^{19}$. Portanto, torna-se necessário aumentar o tempo de reação entre o ácido e a matriz; explicação para o fato das interações entre as variáveis $\mathrm{T}$ e $\mathrm{t}$ para TOC e $\mathrm{V}_{\mathrm{am}}, \mathrm{V}_{\mathrm{Ac}}$ e $\mathrm{t}$ para TC terem mostrado tendência em serem significativas ao sistema, uma vez que têm-se conhecimento de que maiores valores de ácido e de temperatura permitem maior eficiência da digestão polimérica.

Com os resultados obtidos foi possível obter uma modelagem matemática significativa para o TC, $\operatorname{com} p=0,0144$ para o modelo e $p=0,2670$ para a falta de ajuste. Já para o TOC, obteve-se $p=0,0156$ para o modelo e $p=0,5330$ para a falta de ajuste. Os coeficientes de determinação $\left(\mathrm{R}^{2}\right)$ para TC e TOC foram 0,9762 e 0,9752 , respectivamente. $\mathrm{O}$ mesmo não foi observado para as respostas de carbono inorgânico, o que pode ser justificado pela insignificância dos valores dessa resposta frente às concentrações de TC e TOC. E, em virtude disto, adotou-se a concentração de carbono total para o estudo da superfície de resposta.

Com a finalidade de aprofundar o estudo da influência das variáveis e otimizar o sistema de digestão, isto é, obter o menor teor de carbono possível nas amostras após a digestão ácida, realizou-se o planejamento composto central esférico. Este consiste em um conjunto de técnicas estatísticas e matemáticas para o desenvolvimento, melhoria e otimização de processos químicos, físicos, biológicos, entre outros. ${ }^{23-24}$ Esta técnica avalia mais detalhadamente as variáveis significativas obtidas da triagem, por apresentar um número maior de níveis.

O planejamento composto central é um tipo de planejamento para a construção de superfícies de resposta que permite a obtenção de um modelo quadrático. Para o sistema de digestão aqui estudado foi estabelecido um modelo correlacionando a concentração de carbono total com as variáveis volume de amostra, volume de ácido, tempo e temperatura de digestão.

Os ensaios para a análise de superfície de resposta foram realizados aleatoriamente, assim como na triagem. Todas as soluções resultantes de cada ensaio foram analisadas através do analisador de ToC como descrito no item Otimização Multivariada - Triagem, e os dados obtidos estão apresentados na Tabela 4.

Os resultados da metodologia de superfície de resposta para a concentração de carbono total (TC) confirmaram que somente o $\mathrm{V}_{\mathrm{am}}$ (variável 1) é significativo ao sistema $(p=0,0000)$, conforme visualizado na triagem, onde constatou-se o decréscimo no teor de carbono das amostras com o menor nível estudado $(5,00 \mathrm{~mL})$ - efeito negativo. Os erros associados a cada coeficiente foram avaliados pelo teste $t(\alpha=0,05)$, e por meio dos resultados da análise da concentração de carbono total foi possível construir um modelo quadrático por regressão linear múltipla (Equação 1). Observou-se também que o sistema possui comportamento não-linear, pois a interação quadrática da variável $2\left(\mathrm{~V}_{\mathrm{Ac}}\right)$ foi significativa ao sistema $(p=0,0194)$. Na Tabela 5 está apresentada a ANOVA para o modelo obtido.

$$
\begin{aligned}
\mathrm{TC}= & 1,6593( \pm 0,163)+0,6060( \pm 0,058) \times \mathrm{V}_{\mathrm{am}}+ \\
& 0,1651( \pm 0,061) \times \mathrm{V}_{\mathrm{Ac}}{ }^{2}
\end{aligned}
$$

onde $\mathrm{TC}=$ concentração de carbono total, $\mathrm{V}_{\mathrm{am}}=$ volume de amostra e $\mathrm{V}_{\mathrm{Ac}}=$ volume de ácido.

Observa-se pela ANOVA (Tabela 5) que o modelamento matemático proposto pelo planejamento composto central foi uma regressão significativa, ao nível de significância de 0,05 , e que não houve falta de ajuste. O gráfico de valores observados versus valores estimados (Figura 3 ) apresentou-se linear com y $=0,9163 \mathrm{x}+0,1583$ e $\mathrm{R}^{2}=0,9163$.

A Figura 4 (gráfico de resíduos) mostra que os resíduos (diferença entre os valores observados e os valores estimados) estão aleatoriamente distribuídos, confirmando que a falta de ajuste não é significativa para o modelo pois, como esperado, a maior parte dos desvios está relacionada ao erro puro, que é o erro experimental.

A abordagem de otimização multivariada utilizada proporcionou maior média de degradação polimérica, visto que a concentração inicial de carbono total de $4,6154 \mathrm{mg} \mathrm{L}^{-1}$ foi reduzida a $0,5540 \mathrm{mg} \mathrm{L}^{-1}$, ou seja, ocorreu a decomposição de $87,80 \%$ da matriz carbonácea (Figura 5); a taxa média de degradação previamente aos planejamentos experimentais não ultrapassava $12,00 \%$. 
Tabela 4. Resultados (concentração em $\mathrm{mg} \mathrm{L}^{-1}$ de carbono total) dos ensaios experimentais do planejamento composto central, com três replicatas no ponto central (ensaios 25 a 27), para otimização das condições experimentais da digestão de micropartículas poliméricas: volume de amostra $\left(\mathrm{V}_{\mathrm{am}} / \mathrm{mL}\right)$, volume de ácido $\left(\mathrm{V}_{\mathrm{Ac}} / \mathrm{mL}\right)$, temperatura $\left(\mathrm{T} /{ }^{\circ} \mathrm{C}\right)$ e tempo de digestão ( $\mathrm{t} / \mathrm{min}$ ). Os valores em parênteses representam os níveis decodificados. A última coluna corresponde aos valores de concentração de carbono total (TC) ( $\mathrm{m} \mathrm{L}^{-1}$ ) obtidos para cada ponto experimental do planejamento

\begin{tabular}{lccccc}
\hline Ensaio & $\mathbf{V}_{\mathbf{a m}}$ & $\mathbf{V}_{\mathbf{A c}}$ & $\mathbf{T}$ & $\mathbf{t}$ & $\mathbf{T C}$ \\
\hline $\mathbf{1}$ & $-1(7,5)$ & $-1(7,5)$ & $-1(50)$ & $-1(60)$ & 1,4740 \\
$\mathbf{2}$ & $-1(7,5)$ & $-1(7,5)$ & $-1(50)$ & $+1(120)$ & 1,1720 \\
$\mathbf{3}$ & $-1(7,5)$ & $-1(7,5)$ & $+1(70)$ & $-1(60)$ & 1,2300 \\
$\mathbf{4}$ & $-1(7,5)$ & $-1(7,5)$ & $+1(70)$ & $+1(120)$ & 1,1740 \\
$\mathbf{5}$ & $-1(7,5)$ & $+1(12,5)$ & $-1(50)$ & $-1(60)$ & 1,1130 \\
$\mathbf{6}$ & $-1(7,5)$ & $+1(12,5)$ & $-1(50)$ & $+1(120)$ & 1,1720 \\
$\mathbf{7}$ & $-1(7,5)$ & $+1(12,5)$ & $+1(70)$ & $-1(60)$ & 1,1380 \\
$\mathbf{8}$ & $-1(7,5)$ & $+1(12,5)$ & $+1(70)$ & $+1(120)$ & 1,3530 \\
$\mathbf{9}$ & $+1(12,5)$ & $-1(7,5)$ & $-1(50)$ & $-1(60)$ & 2,8540 \\
$\mathbf{1 0}$ & $+1(12,5)$ & $-1(7,5)$ & $-1(50)$ & $+1(120)$ & 2,1420 \\
$\mathbf{1 1}$ & $+1(12,5)$ & $-1(7,5)$ & $+1(70)$ & $-1(60)$ & 2,1150 \\
$\mathbf{1 2}$ & $+1(12,5)$ & $-1(7,5)$ & $+1(70)$ & $+1(120)$ & 2,2000 \\
$\mathbf{1 3}$ & $+1(12,5)$ & $+1(12,5)$ & $-1(50)$ & $-1(60)$ & 2,5110 \\
$\mathbf{1 4}$ & $+1(12,5)$ & $+1(12,5)$ & $-1(50)$ & $+1(120)$ & 2,5060 \\
$\mathbf{1 5}$ & $+1(12,5)$ & $+1(12,5)$ & $+1(70)$ & $-1(60)$ & 3,2380 \\
$\mathbf{1 6}$ & $+1(12,5)$ & $+1(12,5)$ & $+1(70)$ & $+1(120)$ & 2,5910 \\
$\mathbf{1 7}$ & $-2(5,0)$ & $0(10,0)$ & $0(60)$ & $0(90)$ & 0,5770 \\
$\mathbf{1 8}$ & $+2(15,0)$ & $0(10,0)$ & $0(60)$ & $0(90)$ & 2,6840 \\
$\mathbf{1 9}$ & $0(10,0)$ & $-2(5,0)$ & $0(60)$ & $0(90)$ & 2,6550 \\
$\mathbf{2 0}$ & $0(10,0)$ & $+2(15,0)$ & $0(60)$ & $0(90)$ & 2,1730 \\
$\mathbf{2 1}$ & $0(10,0)$ & $0(10,0)$ & $-2(40)$ & $0(90)$ & 2,1120 \\
$\mathbf{2 2}$ & $0(10,0)$ & $0(10,0)$ & $+2(80)$ & $0(90)$ & 1,4680 \\
$\mathbf{2 3}$ & $0(10,0)$ & $0(10,0)$ & $0(60)$ & $-2(30)$ & 2,2160 \\
$\mathbf{2 4}$ & $0(10,0)$ & $0(10,0)$ & $0(60)$ & $+2(150)$ & 2,2350 \\
$\mathbf{2 5}$ & $0(10,0)$ & $0(10,0)$ & $0(60)$ & $0(90)$ & 1,7610 \\
$\mathbf{2 6}$ & $0(10,0)$ & $0(10,0)$ & $0(60)$ & $0(90)$ & 1,6110 \\
$\mathbf{2 7}$ & $0(10,0)$ & $0(10,0)$ & $0(60)$ & $0(90)$ & 1,6060 \\
\hline & & & & & \\
$\mathbf{1 0}$ & & & & & \\
$\mathbf{1 0}$ & -100 &
\end{tabular}

Tabela 5. Tabela ANOVA para o modelo matemático obtido pelo planejamento composto central para a digestão de partículas poliméricas. $\mathrm{FV}=$ fonte de variação, $\mathrm{SQ}=$ soma quadrática, $\mathrm{nGL}=$ número de graus de liberdade, $F$ calc $=$ valor do teste $F$ calculado, $\mathrm{SG}=$ significativo e $p=$ parâmetro estatístico $p$

\begin{tabular}{|c|c|c|c|c|c|}
\hline FV & SQ & nGL & MQ & Fcalc & $p$ \\
\hline Regressão & 10,5103 & 14 & 0,7507 & 9,3869 & SG 0,0002 \\
\hline Resíduos & 0,9597 & 12 & 0,0800 & & \\
\hline Falta de ajuste & 0,9597 & 10 & 0,0960 & 12,3702 & 0,0770 \\
\hline Erro puro & 0,0155 & 2 & 0,0078 & & \\
\hline Total & 11,4700 & 26 & & & \\
\hline \% variação explicada & & & & 91,63 & \\
\hline \% máximo de variação explicável & & & & 99,86 & \\
\hline
\end{tabular}

Obteve-se como ótimo de digestão as condições do ensaio 17 do planejamento CCD esférico (Tabela 6). Esta foi a condição que propiciou $87,80 \%$ de degradação da matriz polimérica, conforme anteriormente citado.

Os planejamentos experimentais multivariados possibilitaram estabelecer um procedimento que utiliza baixo uso de reagente

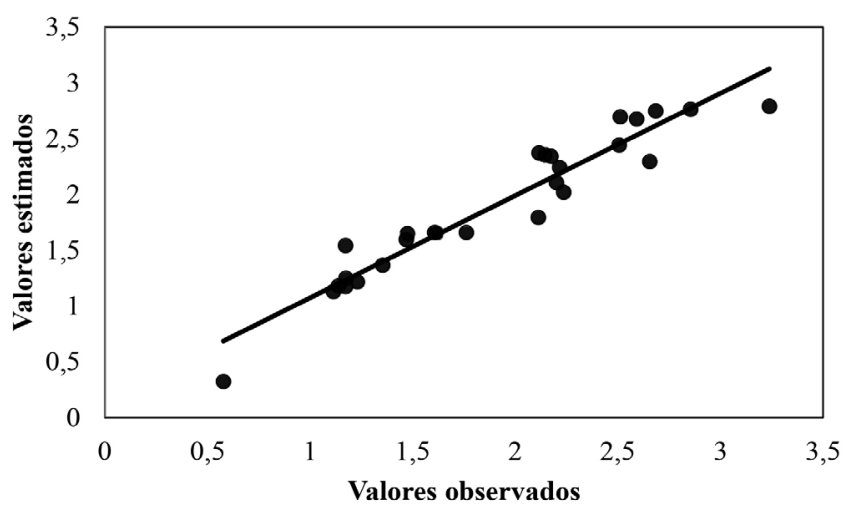

Figura 3. Gráfico de valores observados versus valores estimados para a concentração de carbono total pela metodologia de superfície de resposta

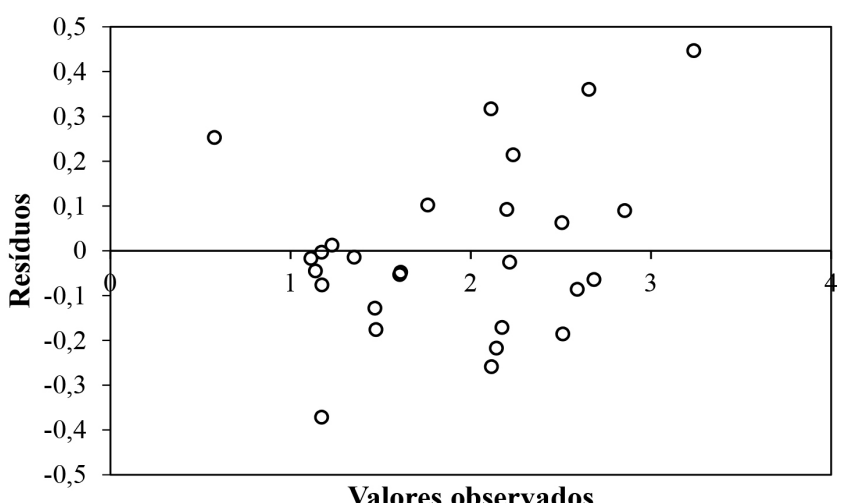

Figura 4. Gráfico de resíduos para a concentração de carbono total na metodologia de superfície de resposta

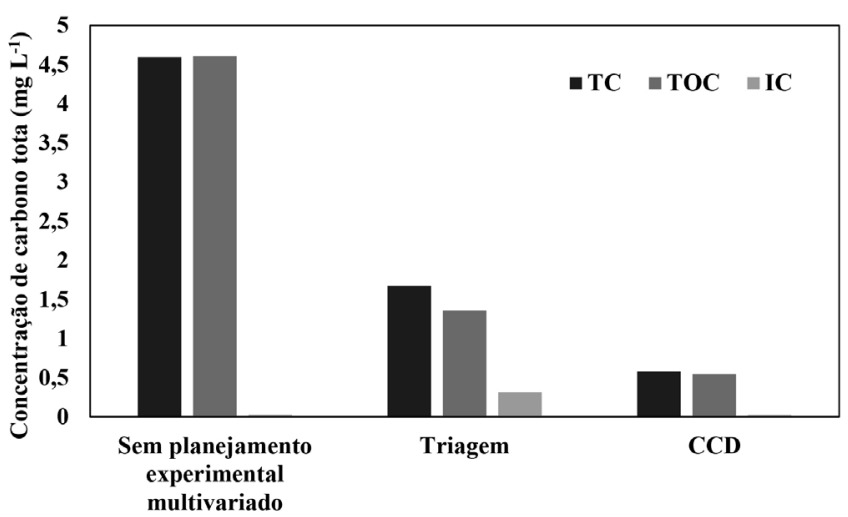

Figura 5. Variação da degradação polimérica entre ensaios sem e com o uso de planejamentos experimentais multivariados. $T C=$ carbono total; $T O C=$ carbono orgânico total $; I C=$ carbono inorgânico e $C C D=$ planejamento experimental composto central

Tabela 6. Condição experimental otimizada para a degradação de carbono de micropartículas poliméricas carreadoras de cátions metálicos de sistemas de liberação controlada

\begin{tabular}{cccc}
\hline $\begin{array}{c}\text { Volume de } \\
\text { amostra }\end{array}$ & $\begin{array}{c}\text { Volume de } \\
\text { ácido }\end{array}$ & Temperatura & $\begin{array}{c}\text { Tempo de } \\
\text { digestão }\end{array}$ \\
\hline $5,00 \mathrm{~mL}$ & $10,00 \mathrm{~mL}$ & $60^{\circ} \mathrm{C}$ & $90 \mathrm{~min}$ \\
\hline
\end{tabular}

(ácido) e é relativamente rápido; características necessárias para um processo de preparo de amostras ideal ${ }^{19}$, tendo sido possível obter um método de digestão de amostras adequado, simples e de baixo 
custo para o preparo de micropartículas poliméricas carreadores de cátions metálicos para análise por FAAS.

\section{CONCLUSÃO}

Os sistemas de liberação micropolímeros carreadores de cátions metálicos essenciais paras as plantas aqui estudados são inéditos e, portanto, estudos relacionados a eles são inexistentes. Assim, é de suma importância a obtenção de procedimentos com condições ótimas para a avaliação da eficiência desta matriz. A metodologia proposta por este trabalho mostrou-se eficaz para a degradação de matrizes poliméricas de sistemas de liberação controlada carreadoras de cátions metálicos, utilizando um sistema de digestão convencional e simples, por meio do uso de ácido e chapa aquecedora e agitadora. Os planejamentos experimentais multivariados permitiram obter uma condição ótima, onde proporcionou-se a quase completa degradação da matriz orgânica. Tal degradação se mostrou eficiente para garantir a qualidade das análises e o não comprometimento do equipamento, por evitar a formação de depósitos de carbono. Além disso, conseguiu-se alcançar menores valores de volume de reagentes concentrados e de temperatura de digestão para sistemas que ainda não são descritos na literatura.

\section{AGRADECIMENTOS}

À Rede Mineira de Química (RQ-MG) (FAPEMIG CEX - RED00010-14), ao Conselho Nacional de Desenvolvimento Científico e Tecnológico (CNPq) (Projeto - 479273/2013-8), à Fundação de Amparo à Pesquisa de Minas Gerais (FAPEMIG) e à Pró-Reitoria de Pesquisa e Pós-Graduação (PROPP) da Universidade Federal de Ouro Preto (UFOP) pelo apoio financeiro e bolsas concedidas. Em especial, os autores também expressam seus agradecimentos ao Laboratório de Química Tecnológica e Ambiental (LQTA) / UFOP, sob a coordenação do professor Dr. Sérgio Francisco de Aquino.

\section{REFERÊNCIAS}

1. Epstein, E.; Bloom, A. J.; Nutrição mineral de plantas: princípios e perspectivas, $2^{\mathrm{a}}$ ed., Londrina: Editora Planta, 2006.

2. Fontes, P. C. R.; Hortic. Bras. 2014, 32, 247.

3. Brasil. Decreto $n^{\circ} 4.954$ de 14 de janeiro de 2004. Dispõe sobre a inspeção e fiscalização da produção e do comércio de fertilizantes, corretivos, inoculantes ou biofertilizantes destinados à agricultura, e dá outras providências. Ministério da Agricultura, Pecuária e Abastecimento, Rio de Janeiro, 2004. 35 p.
4. Lezcano, M.; Al-Soufi W.; Novo M.; Rodríguez-Núñez E.; Tato J. V.; J. Agric. Food Chem. 2002, 50, 108.

5. Manolikar, M. K.; Sawant, M. R.; Chemosphere, 2003, 51, 811.

6. Grillo, R.; Dissertação de Mestrado, Universidade Estadual de Campinas, Brasil, 2011.

7. Grillo, R.; Pereira A. E.; Nishisaka C. S.; de Lima R.; Oehlke K.; Greiner R.; Fraceto L. F.; J. Hazard. Mater. 2014, 278, 163.

8. Grillo, R.; Pereira dos Santos, N. Z.; Maruyama, C. R.; Rosa, A. H.; de Lima, R.; Fraceto, L. F. J. Hazard. Mater. 2012, 231, 1.

9. Memarizadeh, N.; Ghadamyari, M.; Adeli, M.; Talebi, K.; Ecotoxicol. Environ. Saf. 2014, 107, 77.

10. Sun, C.; Shu, K.; Wang, W.; Ye, Z.; Liu, T.; Gao, Y.; Zheng, H.; He, G.; Yin, Y.; Int. J. Pharm. (Amsterdam, Neth.) 2014, 463, 108.

11. Guo, Y.; Yanga, Q.; Yana, W.; Lia, B.; Qiana, K.; Lia, T.; Xia, W.; Hea, L.; Int. J. Environ. Anal. Chem. 2014, 94, 1001.

12. de Assis, L. M.; Zavareze, E. R.; Prentice-Hernandez, C.; de SouzaSoares, L. A.; Braz. J. Food Technol. 2012, 15, 99.

13. Chopra, M.; Kaur, P.; Bernela, M.; Thankur, R.; Food Control 2014, 37, 158.

14. Natarajan, J. V.; Nugraha, C.; Ng, X. W.; Venkatraman, S.; J. Controlled Release 2014, 193, 122.

15. Li, X.; Yu, Y.; Ji, Q.; Qiu, L.; Nanomedicine-Nanotechnology Biology and Medicine 2015, 11, 175.

16. Pinheiro, A. C.; Bourbon, A. I.; Cerqueira, M. A.; Maricato, É.; Nunes, C.; Coimbra, M. A.; Vicente, A. A.; Carbohydr. Polym. 2015, 115, 1.

17. Cai, K.; He, X.; Song, Z.; Yin, Q.; Zhang, Y.; Uckun, F. M.; Jiang, C.; Cheng, J.; J. Am. Chem. Soc. 2015, 137, 3458.

18. Lobo, F. A.; de Aguirre, C. L.; Silva, M. S.; Grillo, R.; de Melo, N. F. S.; de Oliveira, L. K.; de Morais, L. C.; Campos, V.; Rosa, A. H.; Fraceto, L. F.; Polym. Bull. 2011, 67, 479.

19. Cadore, S.; Matoso, E.; Santos, M. C.; Quim. Nova 2008, 31, 1533.

20. Pereira, J. S. F.; Knorr, C. L.; Pereira, L. S. F.; Moraes, D. P.; Paniz, J. N. G.; Flores, E. M. M.; Knappc, G.; J. Anal. At. Spectrom. 2011, 26, 1849.

21. Sakurai, H.; Noro, J.; Kawase, A.; Fujinami, M.; Oguma, K.; Anal. Sci. 2006, 22, 225.

22. Cho, H. J.; Mysung, S. W.; Bull. Korean Chem. Soc. 2011, 32, 489.

23. Teófilo, R. F.; Ferreira, M. M. C.; Quim. Nova 2006, 29, 338.

24. Neto, B. D. B.; Scarminio, I. S.; Bruns, R. E.; Planejamento e otimização de experimentos. São Paulo: Editora da Unicamp, 1995.

25. Lima, D. R. S.; Baeta, B. E. L.; da Silva, G. A.; Silva, S. Q.; de Aquino, S. F.; Quim. Nova 2014, 37, 827.

26. Souto, E. B.; Severino, P.; Santana, M. H. A.; Polim.: Cienc. Tecnol. 2012, 22, 101 . 